\title{
Universal Ultrasonic Generator for Welding
}

\author{
W. Kardyś*, A. Milewski, P. Kogut and P. Kluk \\ Tele and Radio Research Institute, Ratuszowa 11, 03-450 Warsaw, Poland
}

\begin{abstract}
The paper presents design and parameters of a multifunction ultrasonic generator manufactured by Tele and Radio Research Institute. Efficient usage of ultrasonic welding and cutting technology depends on the ability to precisely control process parameters and tune those parameters to suit particular needs. Ultrasonic generator is responsible for such control and tuning. Presented design incorporates powerful 32 bit Cortex M4 microcontroller with floating point unit which allows using modern signal processing methods to calculate parameters of ultrasonic stack in real time. Calculated parameters, especially impedance of the stack are used to estimate load level and identify phases of the welding process such as melting of the welded material. Precise control of the output signal is possible due to innovative design of the direct digital signal synthesis unit implemented in programmable logic device (CPLD). Resonant inverter topology of the generator front end ensures high electrical efficiency of the device. Designed generator is equipped with various communication interfaces such as USB host and slave, Ethernet, Bluetooth, CAN and RS485. Multiple digital inputs and outputs together with load cell interface, 4-20 mA receiver and $0-10 \mathrm{~V}$ analog input enable the generator to monitor and control entire ultrasonic welding system without the need of a PLC unit.
\end{abstract}

DOI: $10.12693 /$ APhysPolA.124.456

PACS: $81.20 . \mathrm{Vj}, 43.35 .-\mathrm{c}, 43.35 .+\mathrm{d}, 77.65 . \mathrm{Dq}, 77.84 . \mathrm{Cg}, 85.50 .-\mathrm{n}$

\section{Introduction}

Ultrasonic welding is based on the introduction of a certain type of acoustic waves to the welded material. This process is also used in ultrasonic drilling, cutting and cleaning. Typical frequency range used is between $18 \mathrm{kHz}$ and $70 \mathrm{kHz}$. Output power varies from hundreds $\mathrm{W}$ to a few $\mathrm{kW}$. Standard welding system consists of an electrical power supply (generator), and ultrasonic stack consisting of a transducer, booster and sonotrode (horn). The generator is responsible for supplying correct electrical signal to the transducer as well as for process control. Transducer transforms electrical supply signal into mechanical longitudinal vibrations which are transferred to sonotrode and ultimately to the welded material [1]. An example of ultrasonic welding machine is shown in Fig. 1.

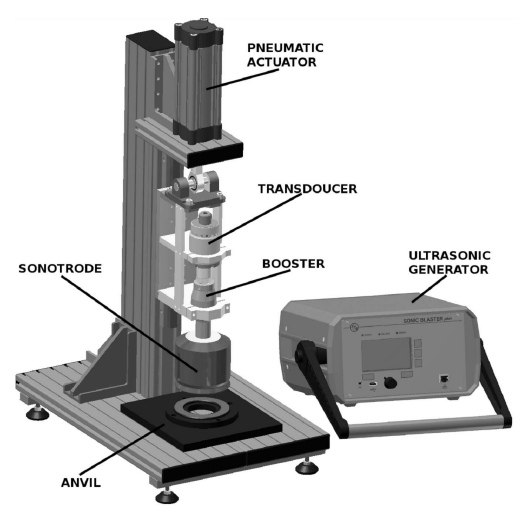

Fig. 1. Simplified model of an ultrasonic welding machine.

*corresponding author; e-mail: witold.kardys@itr.org.pl
The issues related to the design of the ultrasonic generator incorporate the modeling of the electrical part of the system, the development of the topology of the ultrasonic generator, the optimization of its parameters, and the algorithms for the measurement, adjustment and stabilization of the system power.

\section{Ultrasonic technology}

Precise stabilization of the ultrasonic power during the process is essential to maintain high reliability and repeatability of the welds. This is especially important in processing fragile materials, as well as welding small plastic elements and in continuous welding with rotating sonotrode. During the welding process melting and compression of the welded material causes variations in power transfer ratio to the material. In ultrasonic drilling and cutting erosion of the material also causes such variations. Those variations have negative impact on the quality of processed elements. It results in inconsistent weld where some part of the material is damaged by excessive power and some part is welded weakly or not welded at all. To prevent such situations welding system must constantly monitor power delivery level and assure it remains constant.

Especially demanding technology is used in the production process of diamond wire drawing dies. Those elements are used in metal wire manufacturing process. They contain synthetic or natural diamond plates in which precisely dimensioned holes must be drilled. Ultrasonic tools and diamond powder are used in such process. This technology requires very accurate power stabilization - better than $1 \%$ of output power.

\section{Generator power stage}

The main task of the generator is to transmit power to the transducer in such a way that the transducer is 
most efficient at transforming electrical power into mechanical vibration power. Output waveform of ultrasonic generator is sinusoidal and output frequency of particular generator is variable in narrow range. Narrow frequency band and sinusoidal output voltage allow the use of a resonant converter [3]. Such a solution eliminates commutation losses in power switching transistor, hence has better efficiency compared to other converter designs. This approach was used to design ultrasonic generator presented in this article. The unit can deliver to the load up to $500 \mathrm{~W}$ of continuous power in $20 \mathrm{kHz}$ version. Other frequency variants of the device such as $30 \mathrm{kHz}$ and $36 \mathrm{kHz}$ were also developed.

Figure 2 presents a simplified electrical schematic of the resonant converter used in the design. It consists of a half bridge MOSFET switching circuit which generates square signal with variable duty cycle. When the generator is in the first stage of the power control algorithm, in which output voltage is being changed, the duty cycle is between $0 \%$ and $50 \%$.

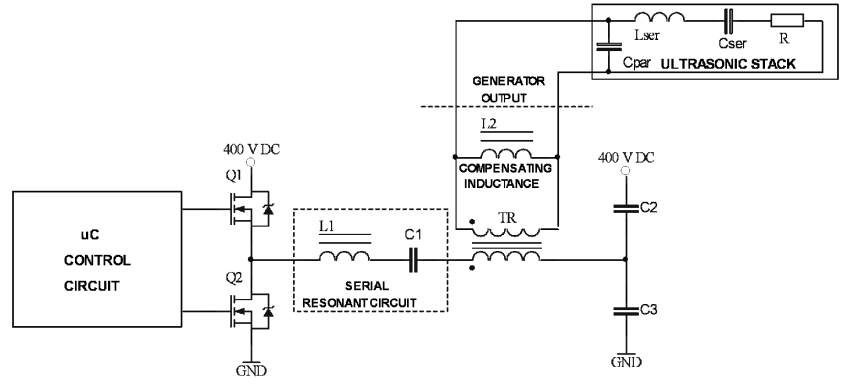

Fig. 2. Simplified electrical schematic of resonant converter.

Signal from the switching circuit is fed to a series resonance circuit. This circuit has a resonance frequency equal to the middle of the frequency range of the generator and low $Q$-factor to allow its proper operation in the whole range. The circuit forms sinusoidal signal transferred to the power transformer which rises output voltage to appropriate level. In presented design the maximum output voltage is $500 \mathrm{~V}$ RMS. There is an additional inductance in the output stage of the generator which is used to compensate the parallel capacitance of the transducer. The compensating inductance and the transducer internal parallel capacitance form a parallel resonance circuit. This circuit has also low $Q$-factor and resonance frequency equal to the middle of the generator frequency range.

\section{Digital control of the generator}

Generation of the control signals for the converter is realized digitally using direct digital signal synthesis technique. Specialized direct digital signal synthesizing unit has been designed for the purpose of presented generator [4]. It was described in the VHDL hardware description language and implemented in MAX II CPLD device. The designed unit is able to generate two complementary square signals with regulated frequency and duty cycle. Output frequency can be programmed up to $100 \mathrm{kHz}$ with $0.1 \mathrm{~Hz}$ resolution.

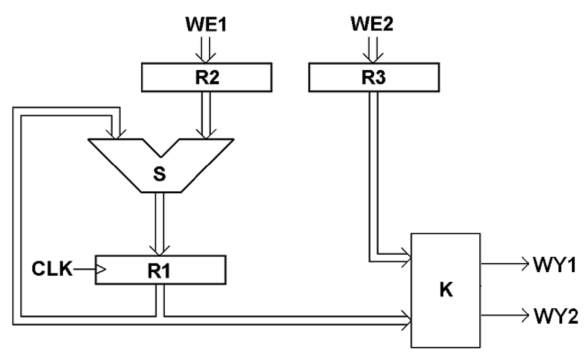

Fig. 3. Diagram of the direct digital signal synthesis block.

Figure 3 presents diagram of the designed block. Parameters of the output signal are digitally measured and processed by the 32 bit ARM Cortex M4 microcontroller which is equipped with a floating point unit (FPU). Built in FPU allows fast and precise calculation of required control signals. This approach is called a digital control loop. The proposed control algorithm sets the output voltage of the generator to a minimum value and the frequency to the value of parallel resonance of the stack. By doing so it ensures minimum power delivery to the stack because, at that point, impedance of the stack is the highest [5]. The second step is to gradually increase the output voltage. If desired output power can be reached by regulating output voltage, the generator keeps output frequency constant and regulates only output voltage to maintain desired power. That, first step of regulation is sufficient only if the desired output power is up to about $10 \%$ to $15 \%$ of the maximum generator output power. In the other case the second step of regulation begins. The generator sets the maximum output voltage and begins to decrease the operating frequency. By doing so it increases the output power because the impedance of the ultrasonic stack decreases if the exciting frequency is decreased from the value of parallel resonance down to series resonance. Maximum electrical power can be delivered at frequency equal to the series resonance point. This technique allows precise control of output power in a wide range of applications and welding methods. Further information about the algorithm can be found in [6] and [7].

\section{Ultrasonic stack diagnostics}

Universal ultrasonic generator must be able to adapt to changes of ultrasonic stack parameters. The device must be able to perform diagnostics of the stack. In the first step the generator has to measure the electrical impedance of the stack as a function of frequency in the frequency range covering operational range of the generator type. For instance, generator designed for $20 \mathrm{kHz}$ 


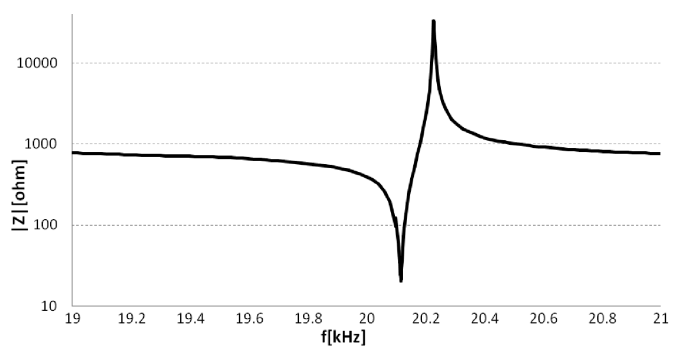

Fig. 4. Electrical impedance module of ultrasonic stack vs. frequency.

technology should operate in the range of $19 \mathrm{kHz}$ up to $21 \mathrm{kHz}$. Example of such measurement is shown in Fig. 4.

The second step is the estimation of the series resonance frequency and parallel resonance frequency of the stack. Those parameters are essential for proper operation of the generator and are used in the process of output power control.

\section{Output power curve}

Digital and precise control over ultrasonic generator output power enables a new method of defining welding process parameters. Presented generator allows the user to predefine the level of the output power at every point in time during the welding process. This approach is called a power curve definition [8]. Precise spot welding of plastic elements often requires variable power delivery during the process.

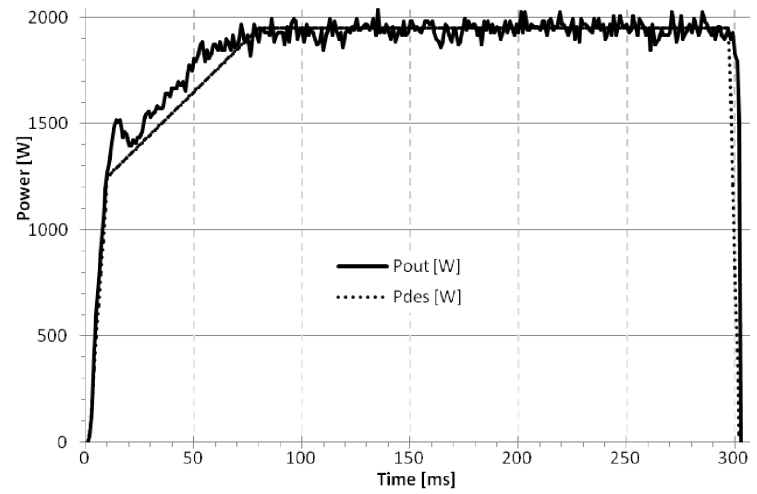

Fig. 5. Output power curve; Pout - real output power of the generator, Pdes - output power curve set by the user.

Figure 5 presents example configuration of the welding process. One of the proposed algorithms of spot welding consists of 4 phases. In the first phase the output power has to raise rapidly to a level at which melting of energy directing notches begins. At that point the power consumption is moderate since the area of contact of both welded elements is small. During the second phase the output power is increased linearly while energy directing notches are melting and the surface area of contact layer between both welded elements increases. The third phase begins when all notches are completely melted and the contact layer surface reaches its maximum area and is no longer increasing. In this phase power delivery is maximum and constant for certain time. The last phase is a rapid linear reduction of power to zero.

\section{Communication interfaces}

The designed generator is equipped with multiple communication interfaces for ease of integration with production lines. It includes wireless Bluetooth interface and USB slave interface for connection with PC computer. USB host interface is used for external memory connection such as a pendrive. Such memory is used to transfer settings and measurement data. RS485 and CAN interfaces are suitable to connect the generator to a control system in the production line. The generator also includes an analog 4-20 $\mathrm{mA}$ current loop receiver and 0-10 V voltage input for connection of external sensors such as transducer temperature sensor. An instrumentation amplifier for an external load-cell has also been included for measuring pressing force of the ultrasonic column. Digital inputs and outputs may be used to control pneumatic actuators in the welding machine as well as to control welding process by the operator. Finally, the generator implements the Ethernet interface with a web page server which allows remote diagnostics and parameter control through Local Area Network.

\section{Conclusions}

New method of controlling output power of the welding system enables the weld quality improvement in various ultrasonic technologies. The ability to flexibly define power levels and its changes during the welding process completes list of process parameters required to efficiently realize every ultrasonic welding technology. Presented ultrasonic generator is suitable for many ultrasonic technologies including spot welding and diamond drilling.

\section{References}

[1] W.P. Mason, Electromechanical Transducers and Wave Filters, 2nd ed., Van Nostrand, New York 1943.

[2] N. Mohan, T.M. Undeland, W.P. Robbins, Power Electronics, 3rd ed., Wiley, USA 2003.

[3] J. Chudorliński, W. Kardyś, Elektronika 51, 99 (2010) (in Polish).

[4] W. Kardyś, P. Kogut, A. Milewski, Elektronika 53, 27 (2012) (in Polish).

[5] P. Kluk, P. Wlazło, Elektronika 50, 70 (2009) (in Polish).

[6] M. Brylski, Elektronika 51, 105 (2010) (in Polish).

[7] P. Kluk, Elektronika 50, 79 (2009) (in Polish).

[8] M. Brylski, Elektronika 51, 102 (2010) (in Polish). 